\title{
Pragmatismo, dogmática jurídica y seguridad nacional: las fuerzas armadas y la inseguridad en América Latina*
}

\section{Adrián Sergio Gimate-Welsh Hernández**}

\begin{abstract}
RESUMEN
La seguridad es una de las problemáticas más apremiantes de México y de América Latina. En la elección presidencial de 2018 en México, la seguridad fue uno de los temas prioritarios del debate de los candidatos presidenciales. En este encuadre, el objetivo de este estudio es dar respuesta a la interrogante, ¿cuál es la normativa que suministra el soporte jurídico al rol que desempeñan las fuerzas armadas en el combate al crimen organizado? Se parte del supuesto de que el objeto de seguridad está constituido de predicaciones de generalidad, de individualidad y de implicaturas en el articulado constitucional que refiere a la seguridad. Este último entiende como un proceso emergente de significación objetiva subjetiva, temporal y contextualmente sensitivo que permite a la normativa constitucional instituir procesos de ensanchamiento o achicamiento hermenéuticos expresados en predicaciones de orden general o individual.
\end{abstract}

\section{PALABRAS CLAVE}

Pragmática, seguridad, objeto de seguridad, significación, individualidad, fuerzas armadas.

\begin{abstract}
Security is one of the most imperative problems in Mexico and Latin America. In the 2018 Presidential Election in Mexico, security was one of the priority topics of the presidential candidates' debate. In this framework, the objective of this study is to answer the question, what is the regulation that provides legal support to the role played by the Armed Forces in battling organized crime? Part of the assumption is that the object of security is constituted by a general predicament of individual implicatures in the Articles of the Constitution that refer to Security. This last concept is understood as an emergent process of objective, subjective, temporal and contextually sensitive significance that allows the Constitutional Norm to institute hermeneutical broadening or shrinking processes expressed in general or individual predicaments.
\end{abstract}

\section{KEYWORDS}

Pragmatic, security, object of security, significance, individuality, armed forces.

*Artículo recibido el 2 de febrero de 2018 y aceptado para su publicación el 30 de agosto de 2018

**Profesor investigador en la Universidad Autónoma Metropolitana - Unidad Iztapalapa, México. (nugo435512@ gmail.com) orcid.org/0000-0003-4562-9403 


\section{SUMARIO}

1. Introducción

2. Modelos pragmático, dogmático y relevancia cognitiva

3. El modelo dogmático jurídico

4. Modelo de la relevancia pragmática

5. Descripción de la dogmática de seguridad

6. Fase interpretativa de la normatividad securitaria

7. Conclusiones

La máxima del pragmatismo es considerar cuáles son los efectos prácticos que se piensan son producidos mediante el objeto de nuestra conceptualización. La concepción de todos esos efectos es la conceptualización del objeto. ${ }^{1}$

\section{Introducción}

Los procesos de significación de todo texto son sucesiones semióticas. ${ }^{2}$ Son tres modos del ser en términos cualitativos, factuales y normativos; son interpretantes -signos- de los procesos que dan sentido a las experiencias del mundo natural y social que en el ámbito de lo jurídico adquieren materialidad en leyes, decretos y reglamentos; operadores deónticos de permisión y de obligación mediante predicaciones de permisión y de obligación. ${ }^{3}$

Desde esta perspectiva, la afirmación sobre el verdadero significado de un concepto o de una proposición coloca a este estudio en el ámbito del pragmatismo peirciano, que distingue entre el objeto de un signo, por una parte, y su significado, por la otra. El primero es la cosa u ocasión, sin considerar qué tan indefinido sea su ámbito de aplicación; el segundo es su significado y sus efectos prácticos que se proyectan mediante mecanismos de implicación. ${ }^{4} \mathrm{El}$ modelo de referencia es el modelo triádico de la significación formulado por Charles Sanders Peirce en sus Collected Papers y en sus Manuscritos.

\footnotetext{
1 Peirce, Charles Sanders, "How to make Our Ideas Clear", en Arthur Burks (ed.), Peirce, Collected papers, Cambrdige, Harvard University Press, 1958. Traducción propia.

2 Peirce, Charles Sanders, "How to make Our Ideas Clear", en Arthur Burks (ed.) Peirce, Collected papers, Cambrdige, Harvard University Press, 1958, p. 16.

3 Burgoa, CARlos, "La deóntica jurídica como clave en la interpetación de las leyes fiscales", Contaduría y administración, núm. 235.

${ }^{4}$ Peirce, Charles Sanders, Collected Papers of Charles Sanders Peirce, Cambridge, Cambridge University Press, 1931.
} 
De manera análoga, se adopta la dogmática jurídica en su vertiente deóntica, en tanto que precisa el qué y el cómo de un sistema jurídico que tiene como dimensiones sustantivas la predicación en su generalidad, en su individualidad y la implicación; como tal, determina o explicita las consecuencias jurídicas que un determinado ordenamiento jurídico asocia con un cierto comportamiento. ${ }^{5}$ Es igualmente un modelo triádico: descripción, interpretación y sistematización.

Dadas las premisas de los modelos anteriores, estos dos modelos se complementan con el modelo de la relevancia pragmática, ${ }^{6}$ acercamiento inferencial a la pragmática que comprende dos principios de relevancia: el cognitivo, que subraya la maximización de la relevancia; y el principio comunicativo que postula la noción de que los enunciados crean las expectativas de maximización de la relevancia. Cabe señalar que el modelo pragmático inferencial tiene como fundamento explicar cómo el oyente o lector infieren el significado a partir de los elementos contenidos en el enunciado. Dicho de otra manera, se trata de un modelo que subraya la relación enunciado-lector/oyente bajo el principio cognitivo y comunicacional.

De acuerdo con las consideraciones previas, el recurso metodológico se sustenta en tres modelos: a) modelo pragmático; $b$ ) modelo dogmático jurídico; y c) modelo de la relevancia pragmática.

\section{Modelos pragmático, dogmático y relevancia cognitiva}

El modelo pragmático es una teoría del significado. En la versión originaria de Peirce, ${ }^{7}$ es el método que permite afirmar el significado verdadero de un concepto, de una doctrina, de una proposición, de una palabra o de un signo. El objeto de un signo es una cosa. Su significado es la idea ligada al objeto por suposición o por afirmación. El supuesto básico del pragmatismo es considerar qué efectos se producen mediante la conceptualización de un objeto. El conjunto de todos esos efectos constituye la concepción completa del objeto en circunstancias posibles o probables. La normativa securitaria exhibe estas características.

Peirce, en las conferencias de 1903, formula lo que denomina las ciencias normativas. En estas, introduce la noción de semiosis como proceso y acción de los signos sobre los interpretantes. En esta línea de pensamiento propone

\footnotetext{
${ }^{5}$ Núñez Vaduero, Álvaro, "Dogmática jurídica", Revista en cultura de la Legalidad, núm. 6.

${ }^{6}$ WIISON, DeIRDRE y SPERBER, DAN, "Teoría de la relevancia", Revista de Investigación Lingüística, vol. 8.

7 Peirce, Charles Sanders, "Pragmatism and Pragmaticism", en Charles Harstshorne and Paul Weiss (eds.), Collected Papers, vol. 5, The Belknap Press of Harvard University Press, Cambridge Mass, 1935, p. 3.
} 
su triada: ${ }^{8}$ primeridad -cualidad-, lo que es sin referencia a ninguna especificidad, categoría de la vaguedad y la originalidad, ámbito de la posibilidad, de lo que puede ser; segundidad -la factualidad-, lo que es en relación con algo más, categoría de la oposición y la diferenciación, lo que es en un contexto particular; y terceridad -la normatividad o ley-, lo que es pero que apela a una segunda entidad en relación con una primera del mismo modo en que se relaciona con la primera y la segunda, categoría de la generalización, del hábito, de la conceptualización y la cognición.

En este encuadre conceptual se encuentra el concepto de seguridad en tanto signo de un objeto o de una cosa aún indescriptible, multidimensional desde otra perspectiva, ${ }^{9}$ pero que puede ligarse a otros objetos por sus cualidades y factualidades. La cualidad o atribución que se le asigne al objeto se establece mediante la relación que el objeto tiene con el signo y a través de este con su interpretante, que es otro signo o significado. Luego entonces, la cualidad que muestra un objeto con otro cualisigno establece otra relación con otro signo que se convierte en su interpretante (legisigno). Así, un interpretante o legisigno puede tener relación con objeto ${ }^{1}$, objeto $^{2}$, objeto ${ }^{3}$, objeto ${ }^{n}$. Igualmente, la cualidad de un objeto, al volverse un interpretante a través de la relación objeto-signo, permite la emergencia. ${ }^{10}$ de un haz de significaciones relacionadas, sea por el hábito, por la experiencia, o por un juicio perceptivo, que es una predicación de generalidad. ${ }^{11}$

\section{El modelo dogmático jurídico}

La dogmática jurídica fija la deóntica de un sistema jurídico, precisa las consecuencias jurídicas de un ordenamiento jurídico vigente, contiene juicios de generalidad y de individualidad $;{ }^{12}$ precisa qué hace y qué debería hacer sobre la base de interpretaciones de implicación del derecho recurriendo a principios, valores, conceptos y teorías dogmáticas. Así, la dogmática jurídica está compuesta de tres planos: $a$ ) descriptivo, $b$ ) interpretativo y c) normativo.

\footnotetext{
${ }^{8}$ Peirce, Charles Sanders, "How to make Our Ideas Clear", en Arthur Burks (ed.) Peirce, Collected papers, Cambrdige, Harvard University Press, 1958.

${ }^{9}$ BuzAn, BarRY, "Peace, Power, and Security: Contending Concepts in the Study of International Relations", Journal of Peace Research, vol. 21, núm. 2.

${ }^{10}$ Quelroz, Joao, EL-Hani, Charbel, "Semiosis as an Emergent Process", A Quarterly Journal of the American Philosophy, núm. 42, vol. 1, pp. 81-84.

11 Peirce, Charles Sanders, Collected Papers of Charles Sanders Peirce, Cambridge, Cambridge University Press, 1931.

${ }^{12}$ Núñez Vaquero, Álvaro, "Dogmática jurídica", Revista en cultura de la Legalidad, núm. 6.
} 
Luego entonces, su quehacer está constituido por el análisis de textos normativos; describe, realiza una interpretación - actividad cognitiva- y sistematiza la interpretación. El carácter descriptivo puede verse sujeto a cuestionamientos según el punto de vista que se adopte: discrecional o ideológico; o racional en términos de la cientificidad o revestido de una perspectiva ético-moral. ${ }^{13}$ Esta tendencia se conoce como la metáfora biológica: describe, interpreta y sistematiza. Pero se homologa con las ciencias médicas en la medida que tiene un fin práctico para un problema específico. Su opuesto sería sostener que carece de cientificidad en la medida en que se sustenta del sentido común; o, desde otra perspectiva, la dogmática revestida de la doxa.

El estudio que aquí se propone se homologa a la metáfora de las ciencias biológicas: contiene una dimensión descriptiva, una fase interpretativa y una de sistematización.

\section{Modelo de la relevancia pragmática}

La teoría de la relevancia es un intento por desarrollar uno de los aspectos sustantivos de Grice: el rasgo esencial de toda comunicación humana, verbal o escrita es la expresión y presencia de una intención. En este marco conceptual se instauran las condiciones para el establecimiento de un modelo de comunicación inferencial. ${ }^{14}$ Lo anterior significa que toda persona que desea comunicar algo proporciona la evidencia lingüística de su intención, lo que permite al hablante o al lector inferir sobre la base de la evidencia de significación proporcionada.

Ahora bien, un enunciado de cualquier tipo está codificado lingüísticamente de modo que su interpretación implica un proceso de decodificación. En este marco, la pragmática inferencial implica explicar cómo el oyente o lector infiere el significado del hablante o del escritor. Para que esto ocurra, el autor del enunciado crea expectativas -huellas lingüísticas- que guían al lector en su actividad de interpretación; esto es, el principio cooperativo y las máximas de calidad (truthfulness), cantidad (informativeness), relación (relevancia) y manera (clarity). Así, la interpretación elegida será la que cumpla con estas máximas del principio de cooperación. Desde la perspectiva de la teoría de la relevancia, el objetivo es explicar en términos cognitivos cuáles son las expectativas de relevancia y cómo contribuyen a la interpretación.

\footnotetext{
${ }^{13}$ Núñez Vaquero, Álvaro, "La cientificidad de la dogmática juridica sobre Carrillo de la Rosa", Revista Telemática de filosofía del derecho, núm. 11, p. 249.

${ }^{14}$ Wilson, Deirdre y Sperber, Dan, "Teoria de la relevancia", Revista de Investigación Lingüistica, vol. 8, pp. 237-286.
} 
Los enunciados generan expectativas de relevancia porque la relevancia es un rasgo cognitivo humano esencial y de acuerdo con esta premisa la teoría de la relevancia adopta el Principio Cognitivo de la Relevancia. ${ }^{15}$ En este marco, la teoría introduce dos nociones: el efecto positivo y la implicación contextual. Bajo estas nociones, un input cognitivo es relevante para una persona cuando su procesamiento genera efectos cognitivos positivos.

Ahora bien, dado que el input son unidades con contenidos codificados en determinados contextos, la interpretación de tales unidades es contexto dependiente o contexto sensible. Pero puede suceder que el significado de una de las unidades del enunciado sea libre de contexto. ${ }^{16}$ Es decir, un término o concepto tiene características principales (core features); la palabra o signo tiene una interpretación constante a través de contextos diferentes. Esta sería la interpretación robusta; o bien, una interpretación débil en la que la interpretación no depende de un contexto. Este es el caso de un concepto comunicado por una palabra que difiere del concepto codificado; y cuando esto sucede, acontece un estrechamiento conceptual: ${ }^{17}$ es decir, se emplea un concepto más específico.

En suma, se trata de un concepto ad hoc que se forma de un concepto estable recurriendo a información asociada con el concepto estable con fines de restricción o ampliación conceptual. Esto sucede en la Ley de Seguridad Interior aprobada en México en 2017 y en los decretos de 2010 y 2011 de Brasil y Argentina que se utilizan para emplear las fuerzas armadas en el combate al crimen organizado.

\section{Descripción de la dogmática de seguridad}

Dada la interrogante planteada al inicio de este estudio sobre cuál es la dogmática jurídica que otorga el soporte a las fuerzas armadas en el combate al crimen organizado, el objetivo de este apartado es describir el contenido de los artículos constitucionales de los países objeto de estudio sobre el concepto seguridad

\footnotetext{
${ }^{15}$ Wilson, DeirdRe y Sperber, Dan, "Teoría de la relevancia", Revista de Investigación Lingüística, vol. 8, p. 251.

${ }^{16}$ Rubio-Fernández, Paula, "Concept Narrowing: The of context independent Information", Journal of Semantics, vol. 25, p. 382.

${ }^{17}$ Carston, Robyn, "Metaphor and Ad Hoc concepts and Word Meaning. More Questions than Answers", University College London. Disponible en: https://www.phon.ucl.ac.uk/publications/WPL/02papers/carston.pdf

WIISON, DIERDRE, "Relevance and Lexical Pragmatics", Italian Journal of Linguistics, vol. 15, núm. 2. Disponible en: https://www.researchgate.net/publication/238625989_Relevance_and_Lexical_Pragmatics
} 
PRAGMATISMO, DOGMÁTICA JURÍDICA Y SEGURIDAD NACIONAL: LAS FUERZAS ARMADAS.

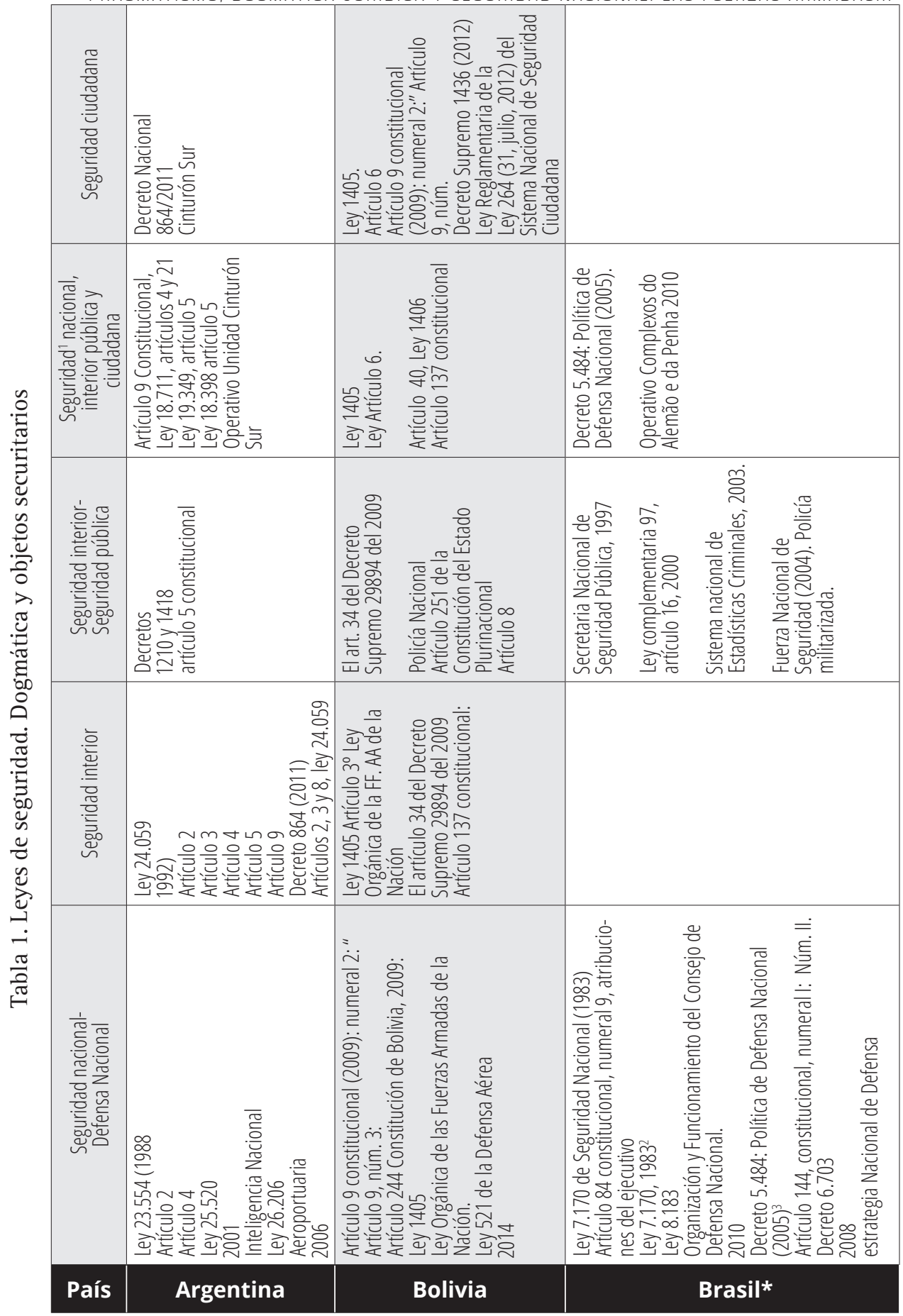


ADRIÁN SERGIO GIMATE-WELSH HERNÁNDEZ

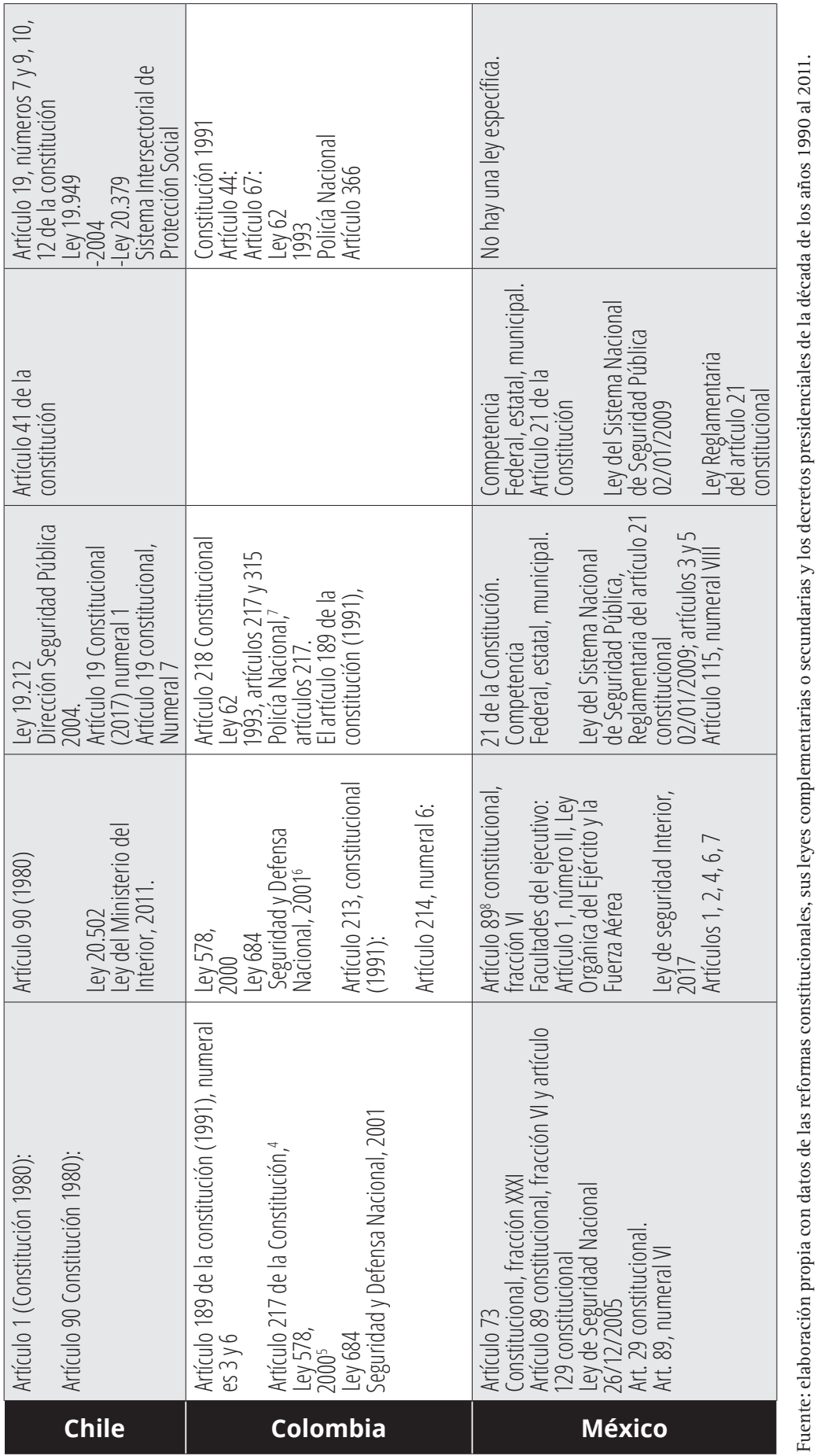




\section{Fase interpretativa de la normatividad securitaria}

La información del articulado constitucional sobre el objeto seguridad en los seis países estudiados muestra cuatro tendencias de conceptualización dogmática jurídica. Como es de todos conocido, el régimen de legalidad radica en que se "puede hacer todo aquello que no está prohibido a contrario sensu". ${ }^{18}$ ¿Y cómo discernir lo que no está permitido? Mediante el análisis de las implicaciones o razonamientos sobre el antecedente y la consecuencia. Esta lógica se relaciona con la perspectiva peirciana.

Los enunciados de los artículos de las constituciones sobre la seguridad refieren a cuatro objetos de seguridad: seguridad nacional o defensa nacional; seguridad interior; seguridad pública y seguridad ciudadana. ¿Cómo se relacionan los objetos de seguridad entre sí en la normativa constitucional? ¿Cuáles son las dimensiones de significación que los distinguen o relacionan? Para responder a estas preguntas, se procede al análisis de la dogmática jurídica desde la perspectiva metodológica enunciada en el párrafo dos, que puntualiza los criterios metodológicos del análisis: el modelo del pragmatismo peirciano, el modelo deóntico o dogmático jurídico y el modelo de la relevancia pragmática. Véase los casos de los países.

\section{a. Argentina}

La ley 23.554 de 1988, artículo 2, define la defensa nacional: "es la integración y la acción coordinada de todas las fuerzas de la nación para enfrentar las agresiones de origen externo”. El rasgo característico de esta norma es la descripción. El enunciado está marcado por la predicación que le imprime el carácter de generalidad. El objeto del signo es una proposición que no especifica, pero que se plantea como una posibilidad. El uso del signo externo, sin embargo, implica dos tipos de objeto con los que el objeto seguridad se relaciona mediante la cualidad de los objetos: Objeto $^{1}$ : otro país; bbjeto $^{2}$ : crimen organizado. Emerge así un proceso de significación que relaciona a los objetos, que a la vez los distingue y los contextualiza. Emerge igualmente el rasgo de factualidad. El cualisigno o primeridad es la multidimensionalidad, la factualidad es la oposición, la diferenciación que, una vez convertida en hábito, norma o ley, deviene un interpretante de la relación de los objetos: otro signo.

\footnotetext{
${ }^{18}$ Rios Martinez, José, "Interpretación en la dogmática jurídica como posibilidad de ciencia del derecho", Revista del posgrado en derecho de la unam, vol. 3, núm. 5.
} 
Ahora bien, la normativa no sólo pone en relación a dos objetos, que da respuesta al qué de la dogmática, sino que propone a la vez el cómo. El sentido de la relación de los objetos es una implicación cognitiva fundada en una experiencia, en un hábito que produce una respuesta no sólo en circunstancias probables, sino también en situaciones posibles. La lógica del enunciado normativo es del tipo

$$
\mathrm{P} \rightarrow \mathrm{Q}
$$

que implica que siempre que se cumpla la condición también acontece la consecuencia. En este caso no es posible el contrario sensu.

Por otro lado, el enunciado normativo satisface el principio cooperativo de la comunicación: la máxima de cantidad se cumple, pues el enunciado normativo contiene la información requerida; la máxima de cualidad igualmente se satisface, dado que el contenido no es falso, el enunciado dogmático está revestido de factualidad; la máxima de relación, que refiere a la relevancia que se sustenta en el principio cognitivo de la relevancia (que genera efectos cognitivos positivos), y puesto que el input cognitivo es relevante, este es robusto, lo cual implica que las características principales de un signo o palabra proyecta una interpretación constante a través de contextos diferentes. Este es el caso de la conceptualización de seguridad como amenazas externas e internas al Estado, lo cual permite una predicación constante en contextos enunciativos distintos.

El artículo 4 de la ley 23.554 es una muestra de la máxima de relevancia, ya que la cualidad del objeto "seguridad nacional" se mantiene en un contexto diferente y en oposición al objeto "seguridad interior" de la ley 23.554; en tanto se establece una distinción, el operador deóntico es de definición, pero también de permisión. Ambos objetos son sensibles contextualmente. A saber, el artículo 4 afirma que "Se deberá tener en cuenta la diferencia fundamental que separa a la defensa nacional de la seguridad interior". En este caso, se tiene una interpretación robusta: el concepto comunicado mantiene el concepto 130 codificado, la interpretación es constante en contextos distintos. Asimismo, se cumple la máxima de manera, ya que el enunciado mantiene la claridad; la intención no es ambigua, es breve y bien secuenciada.

La ley 24.059 argentina de 1992 de seguridad interior está revestida de una predicación: "la seguridad es interior”. Esto le imprime ese rasgo de generalidad y por tanto predica sobre la cualidad de lo doméstico, pero no especifica un referente de individualidad. Sólo con el enunciado siguiente se introduce la factualidad: "resguarda la libertad, la vida y el patrimonio de los habitantes, 
sus derechos, sus garantías y la plena vigencia de las instituciones del sistema representativo" (véase el artículo 2). Así, se encadenan objetos de factualidad.

El artículo 3 de la ley 24.059 muestra una interpretación robusta, en virtud de que en el contexto de la ley de seguridad interior el concepto seguridad nacional mantiene el concepto codificado en otro contexto. Si bien la seguridad nacional se distingue de la seguridad interior en la ley 23.554, en la ley 24.059, en este encuadre mantiene su carga conceptual. El enunciado del artículo 3 dice: "el empleo de los elementos humanos y materiales de todas las fuerzas policiales y de seguridad de la nación". El enunciado "fuerzas policiales y de seguridad de la nación” establece la distinción conceptual, la oposición de objetos diferenciados que relacionan la dogmática securitaria, pero a la vez establece la distinción.

El artículo referido crea el resquicio dogmático para el uso de las fuerzas nacionales en tareas de seguridad interior y seguridad pública. Este es el soporte jurídico que da sustento al decreto 864/2011 empleado en el Cordón Sur de Buenos Aires para contener la violencia, como lo muestra el diagrama siguiente.

Diagrama 2. Argumentación jurídica. ${ }^{19}$ Decreto 864

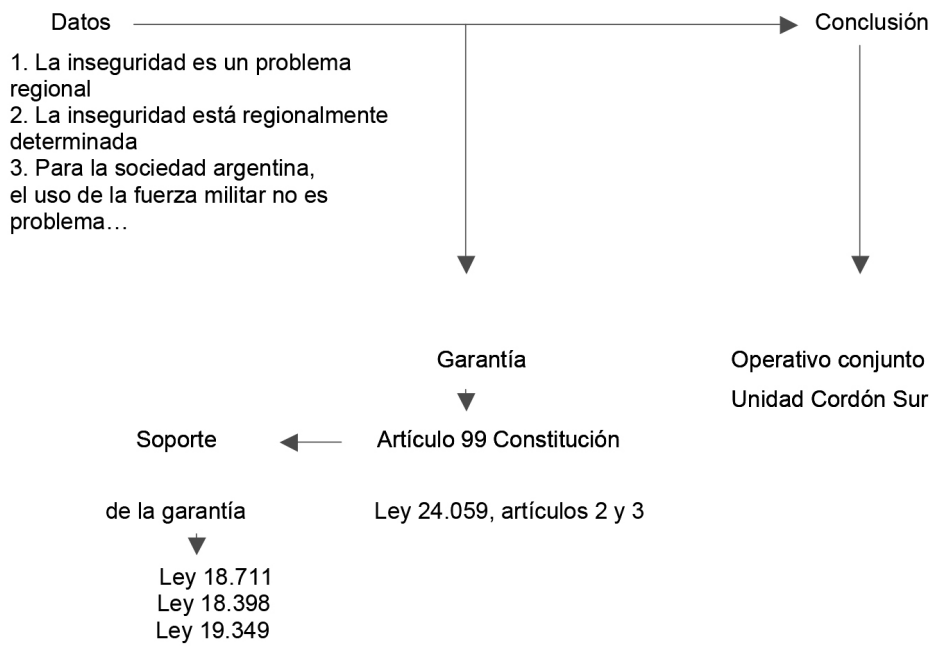

\footnotetext{
${ }^{19}$ Este diagrama es una muestra de la argumentación jurídica subyacente en los decretos empleados para emplear
} las fuerzas armadas en acciones diferentes a la defensa nacional. El caso brasileño muestra una estructura semejante. 


\section{b. Bolivia}

La dogmática jurídica boliviana exhibe semejanzas con la argentina, pero también notorias diferencias. En el caso boliviano, la seguridad nacional está garantizada por el artículo constitucional 244, que trata del rol de las fuerzas armadas; no hay una ley en específico.

Las fuerzas armadas tienen por misión fundamental defender y conservar la independencia, seguridad y estabilidad del Estado, su honor y soberanía del país, garantizar la estabilidad del gobierno legalmente constituido y participar en el desarrollo integral del país.

El objeto seguridad nacional se reviste de las cualidades "independencia" y "soberanía", que se ligan mediante operadores de permisión, que en la norma se manifiestan con operadores descriptivos y atributivos. Dicho de otra manera, la seguridad exhibe dos predicaciones: la seguridad es independencia, la seguridad es soberanía. Es decir, establece una relación con dos objetos. Pero también instaura una correspondencia con dos objetos que corresponden a otra conceptualización de la seguridad de otro contexto, que es el de la seguridad interna: la estabilidad del gobierno y el desarrollo integral del país. Desde este marco, la seguridad muestra relación con tres variables: autodeterminación, estabilidad democrática y desarrollo integral, que es otra predicación. El objeto en cada uno de los casos muestra una proposición de orden genérico: país, soberanía, democracia y desarrollo.

El proceso de significación de los objetos los relaciona, distingue y contextualiza en ámbitos de generalidad e individualidad; transita de la multidimensionalidad a la factualidad mediante la oposición seguridad nacional y seguridad interna mediante la normativa constitucional de artículo 9, numerales 2, 3 y el artículo 244, la Ley 1405 (Ley Orgánica de las Fuerzas Armadas de la Nación) y la Ley 521 (Defensa Aérea). La factualidad queda expresada 132 dogmáticamente en el artículo 34 del Decreto Supremo 29894 del 2009. Esta ley determina que el Ministerio de Gobierno tiene a su cargo formular, dirigir y coordinar políticas para la seguridad pública y el orden público. Así, el objeto seguridad nacional, con sus cualidades y factualidades (core features), mantiene su contenido conceptual en diversos contextos dogmáticos.

Más todavía, la secuencia dogmática del artículo 137 constitucional, que pone en relación a los objetos seguridad nacional y seguridad interna, destaca los rasgos principales de ambos objetos, los opone, pero a la vez establece correspondencias entre ambos. En tanto, el artículo 137 dispone el uso de las 
fuerzas armadas "en caso de peligro para la seguridad del Estado, amenaza externa, conmoción interna, en los casos en que las instituciones legalmente instituidas para este efecto sean insuficientes".

La seguridad nacional como concepto y como objeto se reviste de una interpretación holística, cuyas partes son la seguridad interna, la seguridad pública y la seguridad ciudadana, como se constata asimismo en el artículo 9 constitucional, numeral 2. La lógica enunciativa de la dogmática referida muestra asimismo la implicación siguiente:

$$
\mathrm{P} \rightarrow \mathrm{Q} \text {. }
$$

Acontecido el antecedente, también se cumple la consecuencia; en cuyo caso, no sucede el contrario sensu. El conjunto de enunciados dogmáticos del articulado constitucional satisface el principio cooperativo, pues contiene la información requerida: la información es de calidad y los objetos referidos son de relevancia pragmática desde el punto de vista contextual y cognitivo. La máxima de manera igualmente se cumple pues la intención comunicativa no es ambigua, es breve y bien secuenciada.

La interpretación de los conceptos seguridad nacional, seguridad interna, seguridad pública y ciudadana es robusta, ya que sus rasgos sustantivos (cualidades, factualidades) se mantienen a través de los diferentes contextos dogmáticos. Más todavía, las características principales seguridad nacional y seguridad interna se mantienen en contextos de probabilidad y posibilidad.

\section{c. Brasil}

La dogmática brasileña sobre el objeto de seguridad exhibe semejanzas con la argentina respecto a los casos de excepcionalidad de acuerdo con las atribuciones del ejecutivo para desplegar las fuerzas armadas en tareas de seguridad interior o de seguridad pública. No obstante, en el artículo 84 constitucional, numeral 9, la conceptualización de la seguridad interna está muy relacionada con la seguridad nacional. Como consecuencia, no hay una ley de seguridad interna que la distinga de la seguridad nacional, como es el caso de Argentina.

La distinción que se aprecia entre Estos estados vecinos en parte se atribuye a las diferencias del sistema político y su concreción en la dogmática jurídica. Esta diferencia se aprecia de manera nítida en la descriptiva de la tabla 1. De ahí que el objeto "seguridad interna" se ve subsumido por la "seguridad nacional". La ley 7.170 de Seguridad Nacional (de 1983) prevé los crímenes en contra de la 
seguridad nacional, de la soberanía, del régimen de la democracia representativa y del Estado de derecho; y en contra del tráfico de drogas el artículo 12 de la misma ley.

¿Cómo se predican estos artículos? El artículo 1 "Estado, seguridad y defensa", en el numeral 1.2 define la seguridad según la normativa previa al decreto de ley 5.484 de 2005. Dado que se reforma la dogmática para revestirla de un carácter multidimensional, el objeto de seguridad se reviste de diversas aspectualidades y cualidades de otros objetos securitarios vinculados con la seguridad nacional como respuesta a las nuevas amenazas a las que se enfrentan los Estados. Así, la defensa nacional comprende la seguridad interna, la seguridad pública y la seguridad ciudadana:

Nos primórdios, a segurança era vista somente pelo ângulo da confrontação entre Estados, ou seja, da necessidade básica de defesa externa. À medida que as sociedades se desenvolveram, novas exigências foram agregadas, além da ameaça de ataques externos [...] Gradualmente, o conceito de segurança foi ampliado, abrangendo os campos político, militar, econômico, social, ambiental e outros. Entretanto, a defesa externa permanece como papel primordial das Forças Armadas no âmbito interestata.

El objeto de seguridad nacional objeto ${ }^{1}$ entra en relación con el objeto ${ }^{2}$ : político, objeto ${ }^{3}$ : social, objeto ${ }^{4}$ : económico, objeto ${ }^{5}$ : ambiental, esto es, seguridad interna, pública y ciudadana. Esta relación de objetos se caracteriza por la presencia de operadores deónticos de normas descriptivas.

Esta ley se complementa con el artículo 84 constitucional, numeral 9, sobre las atribuciones del ejecutivo: "decretar estado de defensa y estado de sitio". El artículo 144 constitucional, numerales I y II, definen el qué y el cómo de los sistemas policiales y las fuerzas armadas. Como en los casos de Argentina y Bolivia, la lógica de la dogmática es de la siguiente implicación: al cumplirse el antecedente, se cumple la consecuencia.

El articulado securitario contiene la información necesaria para el sujeto interpretante. Las predicaciones son verosímiles, satisfacen el criterio de factualidad alimentada por el principio cognitivo de relevancia pragmática; la intención comunicativa es clara y bien secuenciada de lo general a lo particular. Se cumple el principio de relevancia cognitiva. 


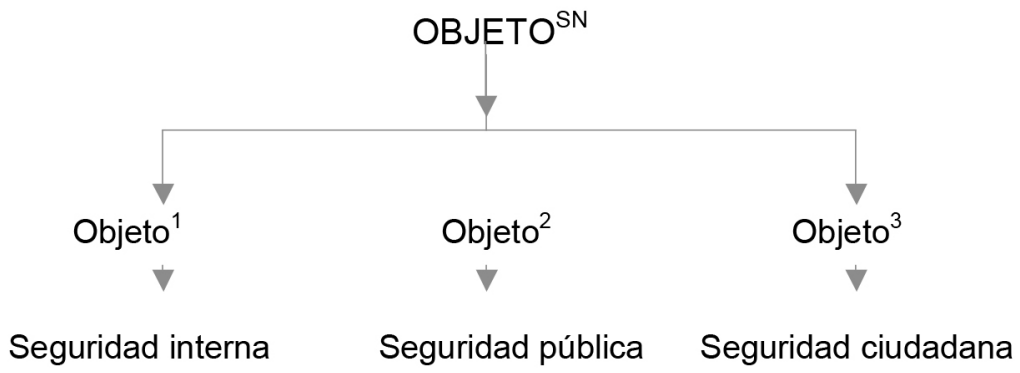

La predicación es la siguiente: la seguridad nacional es seguridad interna, seguridad pública y seguridad ciudadana; la generalidad en sus cualidades y factualidades se relaciona con nuevos interpretantes, que son otros signos. Es una norma descriptiva. Dicho de otra manera, el campo semántico de la seguridad nacional, segmentado en campos semánticos diferenciados por sus cualidades y factualidades, pero que confluyen en el artículo 84, numeral 9 que permite al presidente "decretar el estado de defensa y estado de sitio" que se refuerza con la Ley complementaria 97, artículo 16: "posibilita que las fuerzas armadas intervengan en funciones de defensa civil por decisión del ejecutivo”. No obstante, el Plan Nacional de Seguridad establece que la "seguridad es una actividad pertinente a los órganos estatales y a la comunidad como un todo realizada con el fin de proteger la ciudadanía previniendo y controlando manifestaciones de criminalidad y de violencia”. Ambas dogmáticas jurídicas confluyen en las acciones securitarias, como en el caso de la confluencia de las fuerzas armadas y policiales en el caso del Operativo Complexos do Alemão e da Penha de noviembre, 2010.

\section{d. Chile}

A manera de interpretación implícita, el artículo 1 constitucional (1980) establece la obligación del Estado de "resguardar la seguridad nacional, dar protección a la población y a la familia”, operador deóntico de obligación y atribución. El artículo 19 de la constitución (de 2017) instituye "el derecho a la vida y la integridad física y psíquica de las personas”. Por su parte, el artículo 90 constitucional (de 1980) dispone que "las fuerzas armadas son esenciales para la seguridad nacional y garantizar el orden institucional de la república”.

Ahora bien, ¿qué implica garantizar el orden institucional de la república? La dogmática proyecta como implicatura el "orden democrático interno". Por tanto, el objeto seguridad nacional tiene como cualidad no sólo la generalidad, 
el todo, sino también las partes, cualidades del objeto empírico de interioridad y a su interpretante.

El artículo 90 constitucional (1980) afirma mediante operadores de definición y descriptivos que "las fuerzas del orden y seguridad están integradas sólo por Carabineros y Policía de Investigación [...] que existen para garantizar el orden público y la seguridad pública interior”. La racionalidad jurídica indica que, si el Estado resguarda la seguridad nacional, resguarda también a la población en su carácter general, y a la familia en la singularidad. Esto es, la dogmática exhibe la lógica siguiente: si acontece el antecedente, también lo hace la consecuencia. A saber: $\mathrm{P} \rightarrow \mathrm{Q}$, como lo muestra el encadenamiento dogmático constitucional.

¿Qué proyecta la dogmática de la seguridad nacional de acuerdo con el artículo 1 constitucional? Está compuesto por tres enunciados: resguardo de la seguridad nacional; dar protección a la población; dar protección a la familia, dos enunciados de carácter general y uno individual.

De acuerdo con el pragmatismo peirciano, el efecto producido por los tres objetos de seguridad, en la forma en que estos están encadenados en el artículo 1 de la constitución chilena de 1980, la implicatura es de que el objeto englobante es la seguridad nacional, dada la predicación que se hace de cada uno y de la secuencia discursiva de la dogmática. Es decir, el todo es la seguridad nacional y sus partes son la población y las familias del Estado nación. Es una lógica jurídica del tipo predicación genérica y predicación factual o individualizada.

La conceptualización del objeto seguridad en su cualidad "nacional" se concatena con artículo 41 de la constitución en el que se afirma que "las FF. AA. podrán excepcionalmente ser empleadas en seguridad pública en casos de catástrofes".

El artículo 19 constitucional, numerales 9, 10, 12, a su vez, muestra tres

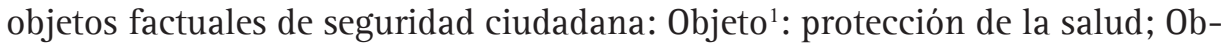
jeto $^{2}$ : derecho a la educación; $0_{\text {bjeto }}^{3}$ : Objeto $^{4}$ : libertad de opinión. Y la ley 19.949 (2004) el Objeto 5 : protección social. Se conjugan objetos relacionados con las libertades civiles y los derechos en los ámbitos del bienestar social, educativo y de salud.

Así, la dogmática jurídica chilena sobre seguridad exhibe tres objetos cuyo articulado constitucional exhibe dos efectos prácticos de significación de conceptualización del objeto securitario: seguridad nacional, seguridad interior y pública y seguridad ciudadana. 


\section{e. Colombia}

La dogmática jurídica colombiana sobre la seguridad conceptualiza cuatro tipos de objetos de seguridad: defensa o seguridad nacional; seguridad interna $u$ orden público; seguridad pública y seguridad ciudadana.

La primera esta enunciada en Ley 684 Seguridad y Defensa Nacional del 2001; en el artículo 217 de la Constitución ${ }^{20}$ (1980): "Las fuerzas militares tendrán como finalidad primordial la defensa de la soberanía, la independencia, la integridad del territorio nacional y del orden constitucional". La ley 684 del 2001, en el artículo 6, define el concepto de defensa nacional. Los operadores deónticos que saltan a la vista son de permiso, de obligación y de atribución.

Es la integración y acción coordinada del Poder Nacional para perseguir, enfrentar y contrarrestar en todo tiempo y cualquier momento, todo acto de amenaza o agresión de carácter interno o externo que comprometa la soberanía e independencia de la Nación, su integridad territorial y el orden constitucional.

Por su parte, el artículo 213 de la constitución de 1991 sobre las facultades del ejecutivo, complementa la dogmática anterior:

En caso de perturbación del orden público [...] que atente contra la estabilidad institucional, la seguridad del estado, o la convivencia ciudadana [...] el Presidente de la República, y que no pueda ser conjurada mediante el uso de las [...] autoridades de policía, con la firma de todos los ministros podrá declarar el estado de conmoción interior. El Presidente informará al legislativo sobre las razones de la declaratoria.

Sobre el artículo anterior, en el marco del equilibrio de poderes, el artículo 214 constitucional, numeral 6, regula que la Corte Constitucional debe recibir de parte del ejecutivo la declaratoria, los decretos legislativos que dicte en el uso de las facultades que dan soporte al decreto presidencial de la declaratoria. Los operadores deónticos son de permisión, de obligación y de atribución.

Respecto a la seguridad pública, el artículo 218 constitucional define las funciones de la policía nacional "su fin primordial es el mantenimiento de [...]

\footnotetext{
${ }^{20}$ Defensa de la soberanía, el territorio, la independencia y el orden constitucional. Constitución Política de Colombia, 2016. Corte Constitucional. Colombia.
} 
los derechos y libertades públicas [...] que los habitantes convivan en paz"; define además el carácter de la policía: "fuerza de carácter civil militarizada".

El alcalde, de acuerdo con el artículo 315, tiene la atribución de "conservar el orden público en el municipio de acuerdo con la ley y las instrucciones que reciba del Presidente de la República y del respetivo gobernador; es la primera autoridad de policía del municipio".

Asimismo, el artículo 44 de la constitución de 1991 establece los derechos fundamentales de los niños, su integridad física, su salud y su seguridad social; el artículo 67 constitucional establece que la educación es un derecho de las personas; y el artículo 366 dispone que "el bienestar general [...] la calidad de la vida de la población son finalidades del Estado, la salud y la educación, saneamiento ambiental y agua potable".

La concatenación dogmática de la conceptualización del objeto de seguridad, en su carácter multidimensional, exhibe relaciones de significación en el articulado constitucional y en las leyes reglamentarias. ${ }^{21}$ En tales relaciones, la totalidad de la significación muestra cómo el proceso de significación y la acción de esta, en el conjunto del articulado constitucional, proyecta un haz de significaciones de objetos de seguridad en que la predicación "la seguridad nacional", en tanto campo semántico, cubre las aspectualidades de sentido del objeto seguridad interior, seguridad pública e incluso la seguridad ciudadana, como establecen los artículos 189, 213, 217 de la constitución, y la Ley 684 del 2001. Esta última subraya la defensa de la soberanía, del territorio, la independencia; pero también el orden constitucional. Es decir, la dogmática, a la vez que afirma la seguridad nacional -soberanía exterior-, tiene como cualidad de significación el campo de significación de la seguridad interna en tanto que refiere a la estabilidad institucional -la soberanía doméstica- así como la convivencia de los ciudadanos.

Estas cualidades del signo "seguridad nacional" instituyen los interpretantes de otros objetos de seguridad, como la seguridad interior, la seguridad pública y la seguridad ciudadana, mediante factualidades del signo defensa nacional que en tanto objetos factuales diferenciados proyectan nuevos interpretantes. Es así como el proceso de significación del articulado de seguridad proyecta la totalidad del concepto de seguridad. La predicación general del objeto "seguridad nacional" desagregada en objeto ${ }^{1}$, objeto ${ }^{2}$, objeto ${ }^{3}$ correspondientes a seguridad interior, seguridad pública y seguridad ciudadana.

\footnotetext{
${ }^{21}$ Ley Reglamentaria de las Fuerzas Armadas, Ley Reglamentaria de la Policía Nacional.
} 
Así, el significado doctrinario de seguridad colombiana, el objeto de seguridad nacional, que es una doctrina, el objeto es el estado nación, se relaciona con otros objetos por implicación o suposición de modo tal que el conjunto de los supuestos constituye la concepción completa en circunstancias probables o posibles; todo ello en el marco de un pragmatismo cognitivo relevante.

\section{f. México}

El caso de la normativa mexicana se parece a los de Brasil y de Colombia, con algunas diferencias, sobre todo a partir de la aprobación de la ley de Seguridad Interior, publicada el 21 de diciembre de 2017. La dogmática securitaria mexicana exhibe procesos de significación cuyo eje se articula del todo a sus partes: de la seguridad nacional a la seguridad interior, a la seguridad pública y la seguridad ciudadana. En otros términos, la seguridad nacional implica la defensa del Estado en tanto predicación general; comprende la defensa de la soberanía externa, la soberanía doméstica o seguridad interior y la soberanía pública.

Así, el artículo 73 constitucional determina que la seguridad es competencia federal, estatal y municipal. A su vez, el artículo 89 constitucional, fracción vi establece:

preservar la seguridad nacional, en los términos de la ley respectiva, y disponer de la totalidad de la Fuerza Armada permanente o sea del Ejército, de la Armada y de la Fuerza Aérea para la seguridad interior y defensa exterior de la Federación.

La atribución de la seguridad a las entidades en el plano multinivel es una predicación general del artículo 73; es factual según el artículo 89 de la constitución: seguridad frente a amenazas externas y seguridad doméstica. En el contexto de la probabilidad, la dogmática prevé el uso del conjunto de las fuerzas armadas (normativa que se refuerza con la ley de Seguridad Interior del 2017, como se verá más abajo). ¿Cuáles son las cualidades del objeto de seguridad en su carácter multidimensional? Son cualidades espaciales, poblacionales y, en la perspectiva de, ${ }^{22}$ son valores democráticos y derechos fundamentales. Por su parte, el artículo 29 constitucional establece:

\footnotetext{
${ }^{22}$ Wolfers, Arnold, "National Security as Ambigous Symbol", Political Science Quarterly, vol. 67, núm. 4.
} 
En los casos de invasión, perturbación grave de la paz pública, o de cualquier otro que ponga a la sociedad en grave peligro o conflicto, solamente el Presidente de los Estados Unidos Mexicanos, con la aprobación del Congreso de la Unión o de la Comisión Permanente cuando aquel no estuviere reunido, podrá restringir o suspender en todo el país o en lugar determinado el ejercicio de los derechos y las garantías que fuesen obstáculo para hacer frente, rápida y fácilmente a la situación.

El artículo 29 establece la normativa del qué y el 89, numeral VI, instituye el cómo:

Preservar la seguridad nacional, en los términos de la ley respectiva, y disponer de la totalidad de la Fuerza Armada permanente o sea del Ejército, de la Armada y de la Fuerza Aérea para la seguridad interior y defensa exterior de la Federación.

El artículo 1, numeral III, de la Ley Orgánica del Ejército y la Fuerza Aérea instituye el uso de las fuerzas armadas en funciones de seguridad nacional y seguridad interior: “"Garantizar la seguridad interior”. El objeto de seguridad pública está instituido en el artículo 21 constitucional y en la ley Reglamentaria del mismo en la Ley Nacional de Seguridad (2009).

La primera establece la responsabilidad multinivel en los términos siguientes:

La seguridad pública es una función a cargo de la Federación, las entidades federativas y los Municipios, que comprende la prevención de los delitos; la investigación y persecución para hacerla efectiva, así como la sanción de las infracciones administrativas, en los términos de la ley, en las respectivas competencias que esta Constitución señala. La actuación de las instituciones de seguridad pública se regirá por los principios de legalidad, objetividad, eficiencia, profesionalismo, honradez y respeto a los derechos humanos reconocidos en esta. Las instituciones de seguridad pública serán de carácter civil, disciplinado y profesional.

La Ley Nacional de Seguridad (2005), por su parte, define la seguridad nacional en los artículos 3, 4 y 5. Las predicaciones respectivas subrayan las amenazas a la "independencia”, el "orden constitucional”, las "instituciones democráticas" 
y la "unidad de las partes de la federación" y la "soberanía" ante amenazas externas, "tráfico de arma" y actos de "delincuencia organizada".

El artículo 115 constitucional, numeral viII establece que "La policía preventiva estará al mando del presidente municipal en los términos de la Ley de Seguridad Pública del Estado" y que "acatará las órdenes que el Gobernador del Estado le transmita en aquellos casos que este juzgue como de fuerza mayor o alteración grave del orden público".

La Ley de Seguridad Interior publicada el 21 de diciembre del 2017, en sus artículos 1, 2, 4, 6 y 7, define la normatividad correspondiente al objeto de seguridad interna en la que se aprecia que el signo -interpretante de un signo- resulta ser la traducción de otro signo -la seguridad nacional- por otro signo que contiene más información. ${ }^{23}$ Así, el artículo 1 de la Ley de Seguridad Interior define el objeto de la manera siguiente: "La seguridad interior es materia de seguridad nacional”. Dicho de otra manera, y por analogía, la seguridad interior cualitativamente es semejante a la seguridad nacional en tanto que esta también se reviste de cualidades espaciales, poblacionales.

Más todavía, comparten cualidades del ámbito democrático como lo expresan los artículos 2 y 7: "preservar los órdenes de gobierno, el orden constitucional, el estado de derecho y la gobernabilidad democrática”, así como "proteger y garantizar protección y garantía de los derechos humanos y sus garantías de conformidad con lo dispuesto por la constitución, los tratados internacionales y los protocolos emitidos por las autoridades correspondientes".

Ahora bien, en la definición conceptual, el artículo 4, numeral Iv, se estipula que la declaratoria de protección a la seguridad interior es condición para que el ejecutivo, al igual que las fuerzas armadas, intervengan de por sí o en coordinación con otras fuerzas y realicen acciones de seguridad interior. Sin embargo, la normatividad del artículo vi contradice el significado del artículo Iv:

Las autoridades federales incluyendo a las Fuerzas Armadas, en el ámbito de sus respectivas competencias, implementarán sin necesidad de Declaratoria de protección a la Seguridad Interior, políticas programas y acciones para identificar, prevenir y atender oportunamente, según su naturaleza, los riesgos contemplados en la Agenda Nacional de Riesgos a la que se refiere el artículo 7 de la Ley de Seguridad Nacional.

\footnotetext{
${ }^{23}$ Peirce, Charles Sanders, Collected Papers of Charles Sanders Peirce, Cambridge, Cambridge University Press, 1931.
} 
De este modo, el signo "interior" es un interpretante de "nacional" en virtud de que comparten cualidades espaciales, poblaciones y valores democráticos. Es un proceso de significación analógico, un proceso metonímico -la parte por el todo y el todo por la parte- que establece correspondencias de significación. El operador deóntico es de permisión y de atribución; un hacer que se permite a las fuerzas armadas.

Identificados los objetos de seguridad en su dimensión conceptual según su dogmática constitucional mexicana, la normativa securitaria instituye el qué de la seguridad -amenazas externas y amenazas internas- y los objetos amenazados -el territorio, la población, las instituciones democráticas-. Para combatir estas amenazas, la dogmática establece que corresponde al sistema policial en el ámbito público y nacional, exterior e interior a las fuerzas armadas.

La predicación normativa es atemporal y probabilística, como lo muestra el uso verbal copulativo, según los artículos 29 constitucional y el 21 de la Ley Nacional de Seguridad. Pero no comprende todas las circunstancias de probabilidad, sobre todo en lo que respecta a las probabilidades de resultados.

La normatividad, tal como se ha expuesto e interpretado, exhibe el realismo político y dogmático: la objetividad social, política y jurídica, en texturas textuales revestidas de racionalidad y objetividad - hechos sociales, políticos, securitarios-. Pero la normatividad basada sólo en generalidades y hechos factuales no es suficiente. ${ }^{24}$ La ley, desde la perspectiva genérica y factual no comprende todas las posibilidades, sobre todo si se considera la multiplicidad de circunstancias probables y posibles. Es decir, la racionalidad jurídica tiene como soporte ciertas circunstancias y ciertos resultados determinados por las circunstancias previstas, pero no comprende muchas otras, sobre todo si se considera que la normatividad tiene una intención, un interés y su correspondiente objetivo, que es el poder.

La hermenéutica securitaria y su expresión en la normativa exhibe un empalme conceptual, objetual; es un encadenamiento tautológico en el que los

142 objetos en su conceptualización empírica son diferentes. El realismo empírico no es el mismo. Se impone así una hermenéutica de primer grado en una segunda hermenéutica de segundo grado, que es la que impera en los países de América Latina y en el caso particular de México.

\footnotetext{
${ }^{24}$ Morgenthau, Hans, Política entre las naciones. La lucha por el poder y la paz, Argentina, Grupo Editor Latino Americano, 1980.
} 


\section{Conclusiones}

Descrita la normativa securitaria de Argentina, Bolivia, Brasil, Chile, Colombia y México, según su articulado constitucional e institucional, y hecha la interpretación de esta de acuerdo con los criterios metodológicos del pragmatismo, de la dogmática jurídica y la relevancia pragmática, se procede ahora a la sistematización de los apartados descriptivo e interpretativo mediante la construcción de un modelo de la normativa de seguridad de los países estudiados con la finalidad. Como se verá, en la propuesta de constructo teórico securitario convergen, de manera implícita, aspectos de los referentes conceptuales expuestos en el apartado metodológico.

El modelo, en tanto constructo, es una representación de un objeto material o mental. Es un signo, desde otra perspectiva. Como tal, representa algo mediante signos que a su vez representan otros signos. El modelo es un patrón de semejanzas, pero también de diferencias que, en conjunto, constituye un objeto tipo, una ley tipo de la que se desprenden leyes tipo.

Así, si un modelo es una representación, es también un signo que provoca sensaciones e imaginaciones; por tanto, está sujeto a interpretaciones alternativas determinadas por relevancias cognitivas alternativas señalizadas por otras circunstancias posibles no previstas en la normatividad. En otras palabras, la normatividad securitaria, si bien está revestida de un realismo político y dogmático, genérico y factual, la racionalidad investida de realismo, también se ve sujeta al signo interpretante determinado por la relevancia cognitiva.

En el encuadre anterior, la normativa de la seguridad en los seis países, de acuerdo con la conceptualización del objeto de seguridad en su multidimensionalidad en los artículos constitucionales de los países estudiados, queda representada del modo siguiente.

El modelo, en tanto representación, es decir, en tanto signo, es un objeto cognitivo. Es un signo que representa una cosa u objeto; es una representación por analogía. Es un signo que genera sensaciones, imaginación; es fuente de procesos de significación. El modelo es un patrón de semejanzas, un objeto tipo que produce "leyes tipo". De ahí las similitudes del articulado constitucional entre los seis países estudiados. Estas semejanzas se manifiestan sobre todo entre México, Colombia, Brasil, Bolivia.

Argentina y Chile exhiben conceptualizaciones del objeto de seguridad que se distinguen de los cuatro países referidos, ya que en el caso argentino la seguridad o defensa nacional se rige por la ley 23.554 (1988) y la seguridad interior por la ley 24.059 (1992), aunque esta ley en sus artículos 3 y 4 establezca 
que la seguridad interior tiene como ámbito el territorio de la República Argentina y para ello se requiera el uso de todas las fuerzas policiales y de seguridad de la nación. La normativa del artículo 99 constitucional se establece como respaldo de los artículos 2 y 3 . El contenido jurídico de estos artículos posibilita el uso de las fuerzas armadas y policiales federales en situaciones de excepción ante la solicitud del gobernador de la provincia.

\section{Diagrama 1. Modelo de seguridad}

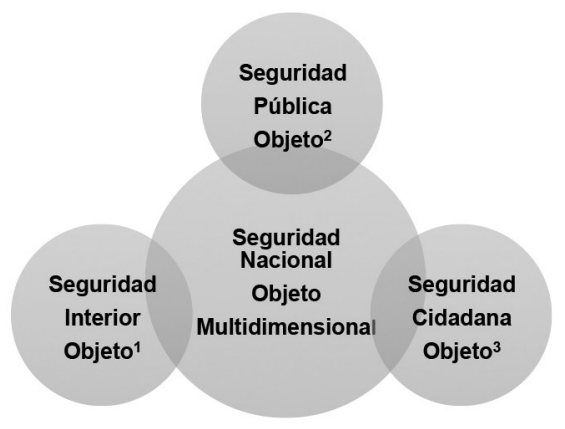

En el caso chileno, la normativa establece que las fuerzas armadas sólo pueden intervenir en la seguridad interior en casos de excepción como los desastres ambientales. Como se ha mostrado en el apartado de la interpretación de la dogmática jurídica, el articulado constitucional sobre la seguridad muestra una argumentación racional sustentada en predicaciones de orden general y factual. Asimismo, el encadenamiento de los artículos exhibe circunstancias probables y posibles. Es, por tanto, una normativa fundada en el realismo político, donde las acciones políticas sustentadas en la intención, el interés y en el poder prevén ciertos resultados o consecuencias anticipadas. Sin embargo, la factualidad o análisis de hechos no es del todo suficiente. Es decir, la factualidad y las acciones que resultan de la aplicación de la normatividad no necesariamente arrojan los resultados esperados.

Las variables circunstanciales y la aplicación de la normatividad pueden arrojar resultados no esperados. El delito y los homicidios no disminuyen en los gobiernos de Felipe Calderón y de Enrique Peña Nieto, por ejemplo. Todo lo contrario, aumentan. El ejemplo más lamentable es el caso de Ayotzinapa, que, de acuerdo con la normativa nacional e internacional, es un caso de genocidio de Estado en el que participaron el ejército, la policía federal y las policías municiapales. Las estrategias de la simulación investigativa y del olvido no son 
aceptables, no sólo por la falta de responsabilidad del Gobierno y de rendición de cuentas al pueblo mexicano, sino por principios éticos, morales y respeto de los derechos fundamentales de los seres humanos. La impunidad es inaceptable en cualquier circunstancia.

La conceptualización del objeto de seguridad en la normatividad constitucional de los estados debería contener esta previsión mediante el articulado de defensa de estos derechos y valores y en la reglamentación de los artículos correspondientes y no limitarse a la finalidad del uso de la autoridad y del poder.

\section{REFERENCIAS}

Burgoa, Carlos, "La deóntica jurídica como clave en la interpetación de las leyes fiscales", Contaduría y administración, núm. 235.

BuzAn, BarRy, "Peace, Power, and Security: Contending Concepts in the Study of International Relations", Journal of Peace Research, vol. 21, núm. 2.

Carston, Robyn, "Metaphor and Ad Hoc concepts and Word Meaning. More Questions than Answers", University College London. Disponible en: https://www.phon. ucl.ac.uk/publications/WPL/02papers/carston.pdf

Morgenthau, Hans, Politica entre las naciones. La lucha por el poder y la paz, Argentina, Grupo Editor Latino Americano, 1980.

NúÑEZ Vaquero, Álvaro, "La cientificidad de la dogmática jurídica sobre Carrillo de la Rosa”, Revista Telemática de filosofía del derecho, núm. 11.

NúÑez Vaquero, Álvaro, "Dogmática jurídica”, Revista en cultura de la Legalidad, núm. 6.

Ordorika, Ana Paula, "El Ejército y la ley", Revista Nexos, 1 de diciembre. Disponible en: http://www.nexos.com.mx/?p=14585

Peirce, Charles Sanders, Collected Papers of Charles Sanders Peirce, Cambridge, Cambridge University Press, 1931.

Peirce, Charles Sanders, "Pragmatism and Pragmaticism", en Charles Harstshorne and Paul Weiss (eds.), Collected Papers, vol. 5, The Belknap Press of Harvard University Press, Cambridge Mass, 1935.

Peirce, Charles Sanders, "How to make Our Ideas Clear", en Arthur Burks (ed.) Peirce, Collected papers, Cambrdige, Harvard University Press, 1958.

QueIroz, Joao, El-Hani, Charbel, "Semiosis as an Emergent Process", A Quarterly Journal of the American Philosophy, núm. 42, vol. 1.

Ríos Martínez, José, "Interpretación en la dogmática jurídica como posibilidad de ciencia del derecho", Revista del posgrado en derecho de la unAM, vol. 3, núm. 5.

Rubio-Fernández, Paula, “Concept Narrowing: The of context independent Information", Journal of Semantics, vol. 25. 
Wilson, Dierdre, "Relevance and Lexical Pragmatics", Italian Journal of Linguistics, vol. 15, núm. 2. Disponible en: https://www.researchgate.net/ publication/238625989_Relevance_and_Lexical_Pragmatics

Wilson, DeirdRe y SPERBER, DAN, "Teoría de la relevancia”, Revista de Investigación Lingüistica, vol. 8.

Wolfers, Arnold, "National Security as Ambigous Symbol”, Political Science Quarterly, vol. 67, núm. 4. 